\title{
Susceptibilidad antimicrobiana en Pseudomona spp., en el Hospital General Docente Cuenca-Ecuador
}

\author{
Antimicrobial susceptibility in Pseudomona spp., in the General Teaching Hospital Cuenca-Ecuador \\ Suscetibilidade antimicrobiana em Pseudomona spp., no Hospital Geral Escola Cuenca-Ecuador
}

\author{
Diana Verónica Barbecho Coraisaca \\ dianisvbarbecho@gmail.com \\ https://orcid.org/0000-0002-2876-8594 \\ Programa de Maestría en Diagnóstico de Laboratorio Clínico y Molecular \\ Universidad Católica de Cuenca-Hospital General Docente, Cuenca-Ecuador
}

Recibido 25 de mayo 2021 / Arbitrado y aceptado 22 de junio 2021 / Publicado 04 de septiembre 2021

\begin{abstract}
RESUMEN
La Pseudomona aeruginosa es un patógeno nosocomial por excelencia; la aparición de cepas multidrogoresistentes (MDR) y extremodrogoresistentes (XDR) cada vez es más frecuente y genera gran preocupación; el uso indiscriminado de antibióticos ha ocasionado esta situación. El objetivo de este estudio fue conocer los diferentes perfiles de resistencia que presentan los aislados objetos de estudio según su procedencia, ya sea a nivel intrahospitalario o de origen comunitario además de caracterizar los principales mecanismos de resistencia y antibiotipos. La presente investigación fue de tipo documental, descriptiva, de corte transversal, para lo cual se utilizó los datos acumulados de susceptibilidad reportados por el departamento de microbiología del Hospital General Docente durante el enero de 2015 hasta diciembre de 2019. Se encontró que en este hospital existe una mayor prevalencia de resistencia a aminoglucósidos (20-25\%), fluoroquinolonas (22-25\%) y carbapenémicos (19-21\%); siendo P. aeruginosa la especie más prevalente. Respecto al tipo de carbapenemasas que son las de interés clínico por su limitante opción terapéutica, se identificó una cepa con fenotipo compatible con metalobetalamasa, se presume que el mecanismo de resistencia predominante fue impermeabilidad. No se identificaron cepas PDR, pero el $21,6 \%$ se mostraron con un perfil MDR, aisladas principalmente en los servicios hospitalarios al igual que las cepas XDR.
\end{abstract}

Palabras clave: Pseudomona; Resistencia antibiótica; Carbapenem; Sensibilidad a los antibióticos

\begin{abstract}
Pseudomonas aeruginosa is a nosocomial pathogen par excellence; the appearance of multidrug resistant (MDR) and extreme drug resistant (XDR) strains is becoming more frequent and is causing great concern; the indiscriminate use of antibiotics has caused this situation. The objective of this study was to know the different resistance profiles presented by the isolated objects of study according to their origin, either at the intrahospital level or of community origin, in addition to characterizing the main resistance mechanisms and antibiotypes. The present investigation was of a documentary, descriptive, cross-sectional type, for which the accumulated susceptibility data reported by the microbiology department of the General Teaching Hospital from January 2015 to December 2019 was used. It was found that in this hospital there is a higher prevalence of resistance to aminoglycosides (20-25\%), fluoroquinolones (22-25\%) and carbapenems (19-21\%); being P. aeruginosa the most prevalent species. Regarding the type of carbapenemases that are of clinical interest due to their limiting therapeutic option, a strain with a phenotype compatible with metallobetalamase was identified, it is presumed that the predominant resistance mechanism was impermeability. No PDR strains were identified, but $21.6 \%$ were shown with an MDR profile, isolated mainly in hospital services as well as the XDR strains.
\end{abstract}

Key words: Pseudomonas; Antibiotic Resistance; Carbapenem; Antibiotic Sensitivity 


\section{RESUMO}

Pseudomonas aeruginosa é um patógeno nosocomial por excelência; o aparecimento de cepas multirresistentes (MDR) e extremamente resistentes aos medicamentos (XDR) está se tornando mais frequente e causando grande preocupação; o uso indiscriminado de antibióticos tem causado essa situação. O objetivo deste estudo foi conhecer os diferentes perfis de resistência apresentados pelos objetos isolados de estudo de acordo com sua origem, seja em nível intra-hospitalar ou de origem comunitária, além de caracterizar os principais mecanismos de resistência e antibióticos. A presente investigação foi do tipo documental, descritiva, transversal, para a qual foram utilizados os dados de suscetibilidade acumulada reportados pelo departamento de microbiologia do Hospital Geral Universitário no período de janeiro de 2015 a dezembro de 2019. Verificou-se que neste hospital há maior prevalência de resistência aos aminoglicosídeos (20$25 \%$ ), fluoroquinolonas (22-25\%) e carbapenêmicos (19$21 \%$ ); sendo $P$. aeruginosa a espécie mais prevalente. Em relação aos tipos de carbapenemases de interesse clínico devido à sua opção terapêutica limitante, foi identificada uma cepa com fenótipo compatível com metalobetalamase, presume-se que o mecanismo de resistência predominante foi a impermeabilidade. Nenhuma cepa PDR foi identificada, mas 21,6\% mostraram ter um perfil MDR, isolado principalmente em serviços hospitalares, bem como as cepas XDR.

Palavras-Chave: Pseudomonas; Resistência a Antibióticos; Carbapenem; Sensibilidade a Antibióticos

\section{INTRODUCCIÓN}

El género Pseudomona se adapta fácilmente al ambiente hospitalario por su ubicuidad y su gran capacidad para colonizar ambientes húmedos. Es responsable de infecciones oportunistas, catalogado como el patógeno intrahospitalario por excelencia. Si bien su prevalencia es menor frente a Enterobacterias como: Klebsiella pneumoniae - Escherichia coli, es alarmante el rápido desarrollo y trasmisión de mecanismos de resistencia (1).
La frecuencia en el aislamiento varía de un hospital a otro, incluso en el mismo hospital entre diferentes salas, las especies con menor frecuencia $P$. stutzeri, $P$. fluorescens, $P$. pútida, entre otras. Sin embargo, $P$. aeruginosa es la especie más relevante y con mayor prevalencia en infecciones nosocomiales, siendo estas las de peor pronóstico. Este patógeno se asocia a altas tasas de mortalidad en la Unidad de Cuidados Intensivos ( $\mathrm{UCl}$ ), tanto en población adulta y pediátrica, así como en pacientes inmunocomprometidos $(2,3)$.

En los últimos años $P$. aeruginosa ha sido ampliamente descrita; posee varios factores de virulencia, siendo el más estudiado la percepción de quórum o Quorum sensing; este factor es clave, para la colonización de dispositivos médicos (puntas de catéter, sondas, entre otros) $(4,5)$. Tiene una estrecha relación con la mortalidad en las $\mathrm{UCl}$ como agente causal de neumonía asociada a la ventilación mecánica (NAV) (6).

Cada vez es más difícil ignorar su amplia distribución con la aparición de cepas multidrogorresistentes (MDR) pues $P$. aeruginosa posee resistencia intrínseca a muchos antibióticos, lo cual limita las opciones terapéuticas $(7,8)$. Esto es debido a su capacidad selectiva evitando que penetren diversas moléculas a su membrana externa o expulsándolos al ingresar en la célula (Bombas Eflujo); en este contexto, la resistencia adquirida tiene mayor relevancia clínica y epidemiológica, se genera mediante la adquisición de plásmidos, por una transferencia 
horizontal de genes con la consecuente aparición de cepas multirresistentes, llegando a extremo drogorresistentes (XDR) incluso pandrogorresistentes (PDX) $(9,10)$.

La Organización Mundial de la Salud (OMS) coordina varios programas ante esta problemática, es así que en febrero del 2017 publicó su primera lista de «patógenos prioritarios» con el objetivo de guiar, promover la investigación y desarrollo de nuevos antibióticos (11). En este boletín se cataloga como prioridad crítica 1 y dentro de los patógenos ESKAPE (Enterococcus faecium, Staphylococcus aureus, Klebsiellapneumoniae, Acinetobacter baumannii, P. aeruginosa $y$ Enterobacter); los de mayor relevancia son los Bacilos Gram Negativos, resistentes a carbapenémicos y a cefalosporinas de tercera generación, por su fácil diseminación $(12,13)$.

En Europa, el programa de Vigilancia de la resistencia a los antimicrobianos, ubica la $P$. aeruginosa tercero en la incidencia nacional; 19,3 \% fueron MDR y $12,2 \%$ tiene resistencia a imipenem, meropenem o ambos (14). Con un panorama en el período desde el 2006 hasta el 2011, el Sistema de Vigilancia Nacional de China reportó, una resistencia a carbapenémicos del $23.6 \%(15,16)$.

Según el estudio SENTRY, Programa de vigilancia antimicrobiana presente en AsiaPacífico, Europa, América Latina y América del Norte, desde 19997 hasta el 2016; América Latina con una frecuencia del $41,1 \%$, fue la región con mayor porcentaje de fenotipos de $P$. aeruginosa MDR (17-19).
En Norte América en donde la incidencia es mucho menor del $18,9 \%$ la red nacional Seguridad en Salud de los Estados Unidos con el apoyo del Centro para el Control y la Prevención de Enfermedades (CDC), en el 2014 reportó $P$. aeruginosa resistente a carbapenémicos que sintetiza metalobetalactamasas (MBL) del tipo VIM (Metalobetalactamasa codificada por el integrón de Verona) proveniente de Tijuana $(17,19,20)$.

El Instituto de Salud Pública en Colombia, según la Red Nacional de Vigilancia de la Resistencia a los Antimicrobianos, en 2018 reportó: 49,4\% $P$. aeruginosa MDR, resistente a los carbapenémicos en un $28,7 \%$ y proviene de $\mathrm{UCl}$; el genotipo más frecuente fue VIM. En el mismo sentido, Brasil reportó una prevalencia del $11 \%$ a nivel nacional (21).

Ecuador no queda exento a esta problemática; la Red Nacional de Vigilancia y Resistencia Bacteriana de Ecuador (REDNARBEC) en el 2015 reportaron los primeros casos de $P$. aeruginosa MDR cuyos genotipos fueron bla-VIM y bla-IMP. Durante el periodo 2018 la prevalencia de este patógeno a nivel nacional fue el $8 \%$ del total de microorganismos enviados a vigilancia; la resistencia a los carbapenémicos en muestras procedentes de hospitalización fue $30 \%$, siendo aún más alarmante en UCl cerca del 50\% (22).

No se tiene un conocimiento preciso de la realidad local, pero existen trabajos de pregrado, en los repositorios de Universidades locales, como el dirigido por Dr. Ochoa médico del servicio de infectología reporto 
una prevalecía del $8,1 \%$ de la $P$. aeruginosa, en pacientes con infecciones asociados a atención de salud (IASS). $(23,24)$.

El conocimiento de los principales mecanismos de resistencia y antibiotipos es fundamental para describir posibles alternativas terapéuticas frente a $P$. aeruginosa. El objetivo de la presente investigación fue caracterizar los principales mecanismos de resistencia y antibiotipos presentes en aislados clínicos de Pseudomona spp., a partir de su prevalencia en el Hospital Vicente Corral Moscoso, periodo 2015-2019.

\section{MATERIALES Y MÉTODOS}

La presente investigación fue de tipo documental, descriptiva, no experimental, de corte transversal con enfoque cuantitativo. La población de estudio estuvo comprendida por 1225 registros en la base de datos que se reportaron con Pseudomona spp., en el Hospital Vicente Corral Moscoso (Hospital General Docente de alta complejidad y centro de referencia de la Zona 6); durante el periodo enero de 2015 a diciembre de 2019.

Criterios de inclusión. Se tomaron en cuenta los registros de todos los cultivos positivos para Pseudomona spp provenientes de diferentes servicios, tanto ambulatorios como hospitalizados.

Criterios de exclusión. Se excluyeron del estudio las cepas provenientes de cultivos de vigilancia epidemiológica, y aquellas que tengan datos incompletos; para la categorización de perfiles de resistencia se excluyeron aislados duplicados.
Los datos fueron descargados y registrados enel programaEpiCenter-BD del departamento Microbiología, luego exportados a Excel® para su posterior análisis; además se complementó con los subregistros de trabajo diario de las pruebas fenotípicas como: inactivación, pruebas de disco combinado y doble disco, con inhibidores específicos (sinergia).

Se utilizó estadística descriptiva, análisis de frecuencia y asociación; para los cuál se generó una base de datos en el programa SPSS versión 20.0 y para al análisis de susceptibilidad e informe acumulado, se utilizó el programa WHONET 5.6; siguiendo las recomendaciones de CLSI.

\section{Aspectos Éticos}

Esta investigación se fundamentó en los principios éticos de la Declaración de Helsinki Adendum de Taiwán 2016; se protegió la privacidad de los pacientes de los cuales se obtuvieron los aislados bacterianos objetos de estudio; fueron manejados con estricta confidencialidad y su información se utilizó solo con fines investigativos.

\section{RESULTADOS Y DISCUSIÓN}

En el hospital, dentro de los gérmenes Gram negativos aislados durante el periodo enero 2015 y diciembre de 2019, la $P$. aeruginosa ocupa el tercer y cuarto lugar en frecuencia de aislamientos. En el servicio de microbiología se analizaron 678 aislados compatibles al género Pseudomona, procedentes predominantemente en el género masculino $(x 2=21,239 p=0,000)$ y del servicio 
de hospitalización $(X 2=220,814 p=0,000)$; se incluyeron las especialidades de cirugía, clínica y ginecología dónde la especie $P$. aeruginosa es más frecuente $(x 2=3242,145 p=0,000)$, tal como se resume en la Tabla 1.

Tabla 1. Características generales de la población de estudio $n=678$.

\begin{tabular}{|c|c|c|}
\hline Sexo & Aislados & Porcentaje \\
\hline Masculino & 399 & $59 \%$ \\
\hline Femenino & 279 & $41 \%$ \\
\hline Servicio & Aislados & Porcentaje \\
\hline Hospitalización Adultos & 227 & $33,5 \%$ \\
\hline Consulta externa & 129 & $19,0 \%$ \\
\hline Pediatría & 130 & $19,2 \%$ \\
\hline Emergencia & 107 & $15,8 \%$ \\
\hline Unidad de Cuidados Intensivos & 85 & $12,5 \%$ \\
\hline Germen aislado & Aislados & Porcentaje \\
\hline Pseudomona aeruginosa & 615 & $90,7 \%$ \\
\hline Pseudomona spp. & 29 & $4,3 \%$ \\
\hline Pseudomona putida & 24 & $3,5 \%$ \\
\hline Pseudomona fluorescens & 7 & $1 \%$ \\
\hline Pseudomona pseudoalcaligenes & 1 & $0,1 \%$ \\
\hline Pseudomona orzyhabitans & 1 & $0,1 \%$ \\
\hline Pseudomona luteola & 1 & $0,1 \%$ \\
\hline
\end{tabular}

En la Figura 1 se representa la tendencia del perfil de susceptibilidad, se destacó que en el año 2017 existió un mayor número de aislados MDR (25,3\%) y XDR (7.79\%).

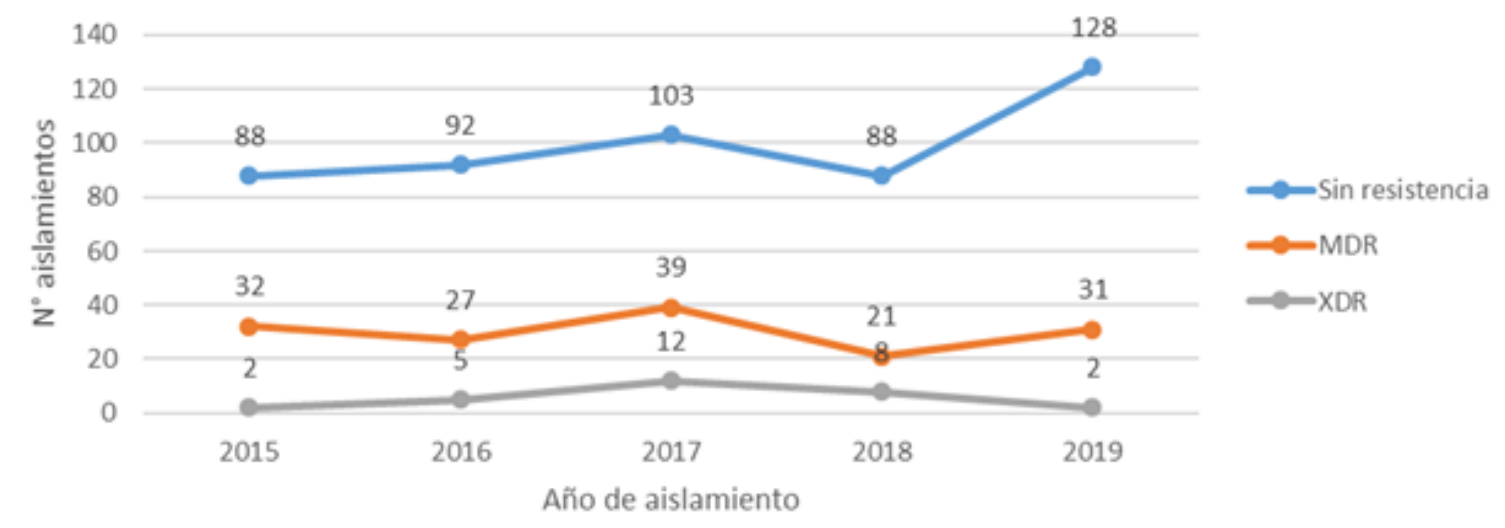

Figura 1. Tendencia de aislamientos según su perfil de resistencia durante el periodo enero 2015 y diciembre 2019. 
En la Tabla 2 se resumen la relación de los fenotipos MDR y XDR según el tipo de muestra y el servicio de procedencia; en donde aquellas $P$. aeruginosa que presentaron resistencia a 3 o más antibióticos se catalogaron como MDR con el $83,6 \%$ mientas que el $16,4 \%$ restante fueron XDR. Por otra parte, se corroboró que no existe relación significativa entre la resistencia a los antimicrobianos y la especie $(x 2=3,123 p=0,210)$. También se correlacionó con el tipo de muestras aquellas catalogadas como secreciones (heridas, hueso, tejido, entre otras) tiene una relación significativa con el fenotipo XDR ( $X 2=40,821 p=0,000)$.

Sin embargo, a pesar del número reducido de aislados en esputo, tracto respiratorio superior $\mathrm{e}$ inferior fueron categorizados como MDR (36,3\%). Los aislados XDR fueron receptadas de los servicios hospitalarios en su mayoría, $(X 2=63,038 p=0,210)$ seguida de la Unidad de cuidados intensivos.

Tabla 2. Relación de aislamiento con cepas Multirresistentes y extremo drogo resistentes $(n=678)$.

\begin{tabular}{lcccc}
\hline \multicolumn{1}{c}{ Germen aislado } & Sin resistencia & $\begin{array}{c}\text { MDR } \\
\mathbf{n = 1 4 7}\end{array}$ & $\begin{array}{c}\text { XDR } \\
\mathbf{n = 2 9}\end{array}$ & Total \\
\hline Pseudomona aeruginosa & $90,4 \%$ & $90,0 \%$ & $100,0 \%$ & $97,3 \%$ \\
Otras especies de Pseudomona & $9,6 \%$ & $10,0 \%$ & $0,0 \%$ & $9,3 \%$ \\
Secreciones & Tipo de muestra & & & \\
Esputo & $33,7 \%$ & $38,0 \%$ & $55,2 \%$ & $35,5 \%$ \\
Orina & $31,1 \%$ & $19,3 \%$ & $0,0 \%$ & $27,1 \%$ \\
Tracto Respiratorio Inferior & $11,8 \%$ & $9,3 \%$ & $24,1 \%$ & $11,8 \%$ \\
Tracto Respiratorio Superior & $8,4 \%$ & $16,7 \%$ & $3,4 \%$ & $10,0 \%$ \\
Sangre & $3,8 \%$ & $7,3 \%$ & $3,4 \%$ & $4,6 \%$ \\
Líquidos biológicos & $3,8 \%$ & $6,0 \%$ & $3,4 \%$ & $4,3 \%$ \\
Punta de catéter & $4,2 \%$ & $1,3 \%$ & $3,4 \%$ & $3,5 \%$ \\
& $3,2 \%$ & $2,0 \%$ & $6,9 \%$ & $3,1 \%$ \\
Hospitalización Adultos & Servicio Hospitalarios & & & \\
Pediatría & $28,7 \%$ & $44,0 \%$ & $62,1 \%$ & $33,5 \%$ \\
Consulta externa & $23,0 \%$ & $10,0 \%$ & $0,0 \%$ & $19,2 \%$ \\
Emergencia & $21,8 \%$ & $13,3 \%$ & $0,0 \%$ & $19,0 \%$ \\
Unidad de Cuidados Intensivos & $16,8 \%$ & $14,7 \%$ & $3,4 \%$ & $15,8 \%$ \\
\hline & $9,6 \%$ & $18,0 \%$ & $34,5 \%$ & $12,5 \%$ \\
\hline
\end{tabular}

Los niveles de concentración inhibitoria mínima (CMI) de $\mathrm{CMI}_{50}$ y $\mathrm{CMI}_{90}$, permanecieron estables durante enero de 2015 y diciembre de 2019 para: ciprofloxacina (0,5-4), gentamicina e imipenem(2-16); dentro de los carbapenémicos: meropenem frente a imipenem fue más estable pues su $\mathrm{CMI}_{50}$ fue menor, como se muestra en la Tabla 3, y lo demuestra en los datos acumulados de resistencia observados en la Figura 2. 
Tabla 3. Susceptibilidad de Pseudomonas aeruginosa

\begin{tabular}{|c|c|c|c|c|c|c|c|}
\hline AÑo & Antibiótico/MIC & $\mathbf{N}$ & $\% R$ & MIC50 & MIC90 & Media & MIC Rango \\
\hline 2015 & \multirow{5}{*}{$\begin{array}{l}\text { Aztreonam } \\
\mathrm{S}<=8 \mathrm{R}>=32\end{array}$} & 53 & 35,8 & 16 & 32 & 11,24 & $1-64$ \\
\hline 2016 & & 99 & 39,4 & 8 & 32 & 11,513 & $1-64$ \\
\hline 2017 & & 82 & 42,7 & 8 & 32 & 13,062 & $2-32$ \\
\hline 2018 & & 92 & 32,6 & 8 & 32 & 11,399 & $2-32$ \\
\hline 2019 & & 56 & 35,7 & 16 & 32 & 12,804 & $2-32$ \\
\hline 2015 & \multirow{5}{*}{$\begin{array}{c}\text { Cefepime } \\
\mathrm{S}<=8 \mathrm{R}>=32\end{array}$} & 46 & 21,7 & 8 & 32 & 6,478 & $1-32$ \\
\hline 2016 & & 73 & 12,3 & 4 & 32 & 5,268 & $1-32$ \\
\hline 2017 & & 133 & 17,3 & 4 & 32 & 5,975 & $1-32$ \\
\hline 2018 & & 102 & 8,8 & 4 & 16 & 5,468 & $1-32$ \\
\hline 2019 & & 64 & 1,6 & 4 & 16 & 4,177 & $1-32$ \\
\hline 2015 & \multirow{5}{*}{$\begin{array}{l}\text { Ceftazidima } \\
\mathrm{S}<=8 \mathrm{R}>=32\end{array}$} & 19 & 15,8 & 4 & 32 & 4,149 & $1-32$ \\
\hline 2016 & & 78 & 16,7 & 4 & 32 & 4,907 & $1-64$ \\
\hline 2017 & & 133 & 18,8 & 4 & 32 & 5,11 & $1-32$ \\
\hline 2018 & & 103 & 22,3 & 4 & 32 & 5,236 & $1-32$ \\
\hline 2019 & & 64 & 9,4 & 4 & 16 & 3,789 & $1-32$ \\
\hline 2015 & \multirow{5}{*}{$\begin{array}{l}\text { Ciprofloxacina } \\
\mathrm{S}<=1 \mathrm{R}>=4\end{array}$} & 54 & 33,3 & 0.5 & 4 & 1,108 & $0,5-4$ \\
\hline 2016 & & 99 & 25,3 & 0.5 & 4 & 1,028 & $0,5-4$ \\
\hline 2017 & & 132 & 32,6 & 0.5 & 4 & 0,979 & $0,125-4$ \\
\hline 2018 & & 102 & 20,6 & 0.5 & 4 & 0,827 & $0,125-4$ \\
\hline 2019 & & 62 & 16,1 & 0.5 & 4 & 0,8 & $0,125-4$ \\
\hline 2015 & \multirow{5}{*}{$\begin{array}{l}\text { Gentamicina } \\
\mathrm{S}<=4 \mathrm{R}>=16\end{array}$} & 54 & 25,9 & 2 & 16 & 4,052 & $2-16$ \\
\hline 2016 & & 99 & 20,2 & 2 & 16 & 3,502 & $2-16$ \\
\hline 2017 & & 133 & 21,8 & 2 & 16 & 3,604 & $2-16$ \\
\hline 2018 & & 102 & 12,7 & 2 & 16 & 3,153 & $2-16$ \\
\hline 2019 & & 64 & 20,3 & 2 & 16 & 3,513 & $2-16$ \\
\hline 2015 & \multirow{5}{*}{$\begin{array}{l}\text { Imipenem } \\
\mathrm{S}<=2 \mathrm{R}>=8\end{array}$} & 53 & 22,6 & 2 & 16 & 2,531 & $1-16$ \\
\hline 2016 & & 99 & 21,2 & 2 & 16 & 2,779 & $1-16$ \\
\hline 2017 & & 131 & 30,5 & 2 & 16 & 2,912 & $0,25-16$ \\
\hline 2018 & & 98 & 29,6 & 2 & 16 & 3,036 & $0,25-16$ \\
\hline 2019 & & 55 & 14,5 & 2 & 16 & 2,051 & $0,5-16$ \\
\hline
\end{tabular}




\begin{tabular}{llcccccc}
\hline AÑo & Antibiótico/MIC & N & \%R & MIC50 & MIC90 & Media & MIC Rango \\
\hline 2015 & & 43 & 18,6 & 1 & 16 & 1,816 & $1-16$ \\
2016 & Meropenem & 74 & 24,3 & 1 & 16 & 2,057 & $1-16$ \\
2017 & S $<=2$ R $>=8$ & 133 & 27,1 & 1 & 16 & 2,000 & $0,5-64$ \\
2018 & & 99 & 24,2 & 1 & 16 & 1,918 & $0,5-16$ \\
2019 & & 59 & 6,8 & 1 & 4 & 1,295 & $0,5-16$ \\
2015 & & 45 & 11,1 & 8 & 128 & 10,556 & $2-128$ \\
2016 & Piperacillina/ & 73 & 16,4 & 8 & 128 & 12,739 & $2-128$ \\
2017 & Tazobactam & 132 & 22,7 & 8 & 128 & 13,885 & $4-128$ \\
2018 & S<=16 R>=128 & 103 & 12,6 & 8 & 128 & 10,976 & $4-128$ \\
2019 & & 64 & 7,8 & 8 & 64 & 9,828 & $4-128$ \\
\hline
\end{tabular}

N=número; \%R: \% de Resistencia; CMI50, concentración inhibitoria mínima al 50\%; CMI90, concentración mínima inhibitoria al 90\%
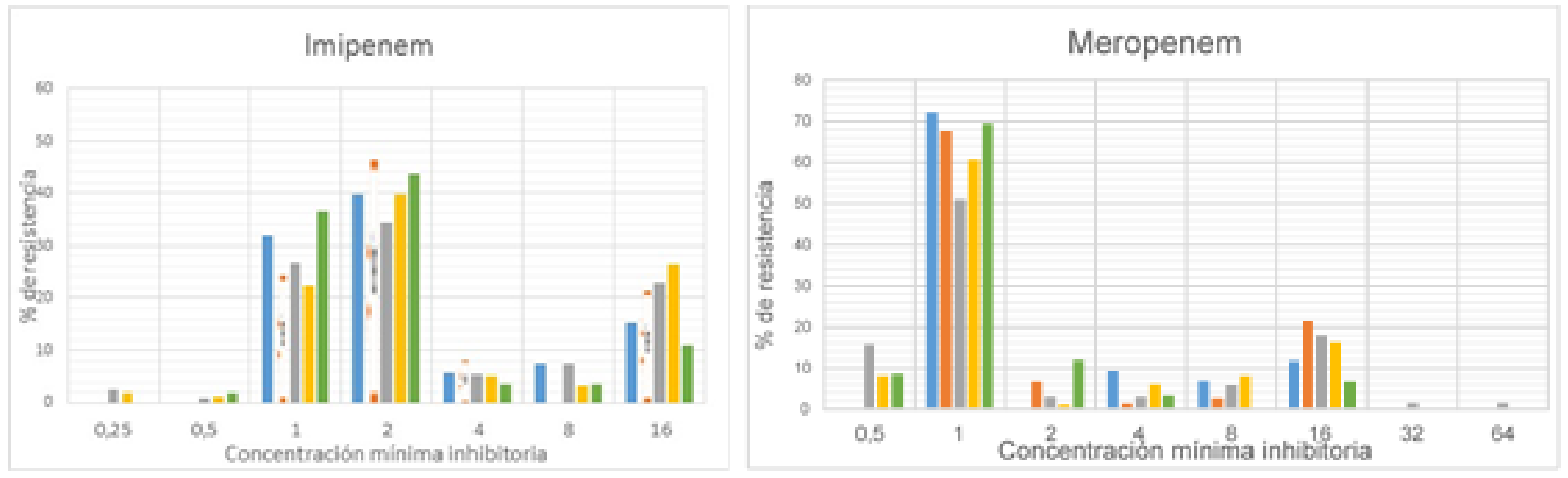

$=2015=2016=2017=2018=2019$

Figura 2. Resistencia anual a imipenem y meropenem en Pseudomona aeruginosa por concentración mínima inhibitoria.

Entre las cefalosporinas, ceftazidima mostró un $81,38 \%$ y cefepime $78,54 \%$ de sensibilidad, en la Tabla 3 se resumen $\mathrm{CMI}_{50^{\prime}}$ y $\mathrm{CMI}_{90}$ misma que es estable. Cefepime muestra una población con tendencia a mayor probabilidad de resistencia como muestra la Figura 3. 


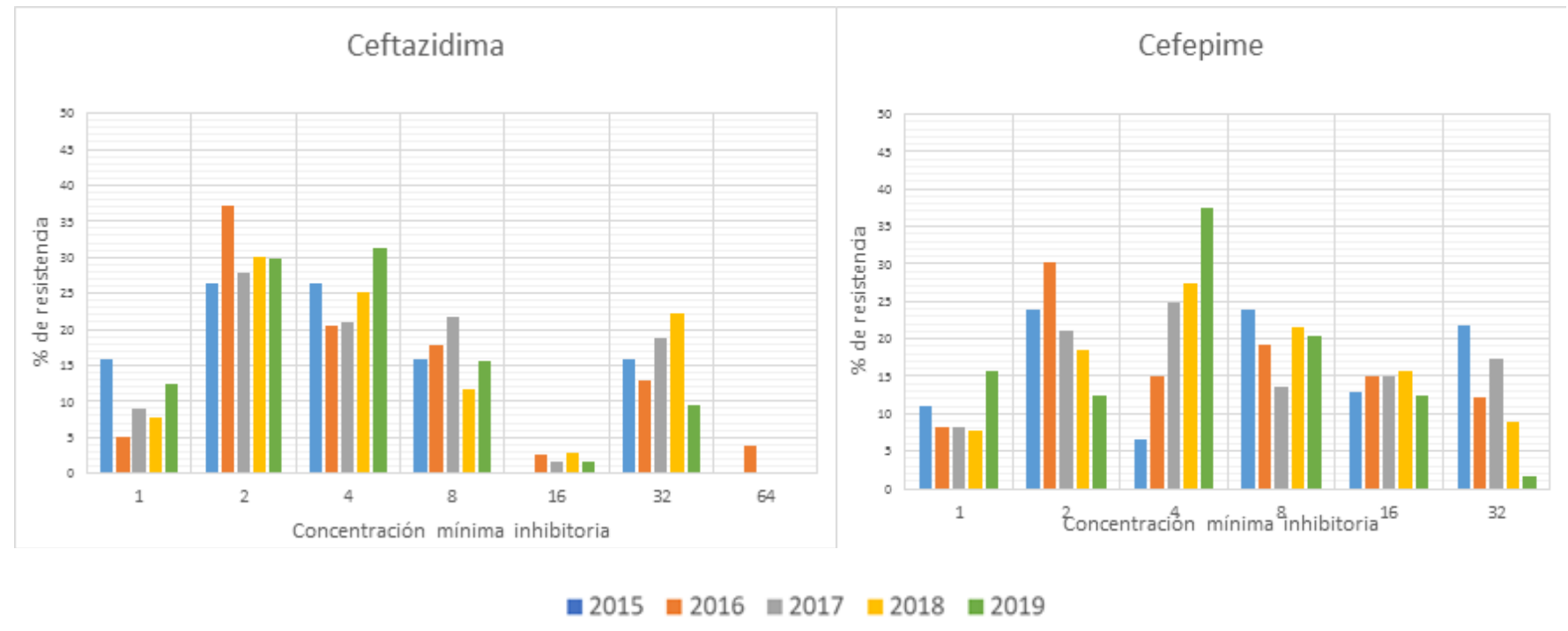

Figura 3. Resistencia anual de ceftazidima y cefepime en Pseudomona aeruginosa por concentración mínima inhibitoria.

Frente a una posible infección por $P$. aeruginosa de acuerdo con el foco infeccioso se puede consultar la Tabla 4 y Tabla 5 , en las cuales se describe el perfil completo de susceptibilidad. Las muestras de orina procedentes de pacientes hospitalizados indicaron que durante el 2017 se aislaron el mayor número de cepas XDR, lo cual se puede evidenciar por un cambio drástico en la cartilla de susceptibilidad y en los 3 primeros años se evidenciaron resistencias críticas; sin embargo, esto dio un giro favorable durante el 2018 y 2019 donde la sensibilidad fue absoluta. 
Tabla 4. Informe acumulado de susceptibilidad de la Pseudomona aeruginosa aisladas en orina en los servicios ambulatorios y hospitalización pacientes del hospital.

\begin{tabular}{|c|c|c|c|c|c|c|c|c|c|c|c|}
\hline Procedencia & Año & $\mathbf{N}$ & ATM \%S & FEP \%S & CAZ \%S & CIP \%S & GEN \%S & IPM \%S & MEM \%S & TZP \%S & AK \%s \\
\hline \multirow{5}{*}{ Ambulatoria } & 2015 & 10 & 85,7 & 100 & 100 & 100 & 100 & 100 & 100 & 100 & 100,0 \\
\hline & 2016 & 10 & 75 & 100 & 87,5 & 75 & 87,5 & 100 & 100 & 100 & 85,7 \\
\hline & 2017 & 11 & 100 & 100 & 100 & 90 & 90 & 88,9 & 100 & 100 & 100,0 \\
\hline & 2018 & 3 & & 33,3 & 66,7 & 100 & 100 & 66,7 & 66,7 & 66,7 & 100,0 \\
\hline & 2019 & 6 & & 83,3 & 100 & 66,7 & 83,3 & 83,3 & 100 & 83,3 & 83,3 \\
\hline \multirow{5}{*}{ Hospitalarias } & 2015 & 8 & 28,6 & 0 & 33,3 & 42,9 & 28,6 & 66,7 & 75 & 0 & 38,8 \\
\hline & 2016 & 11 & 44,4 & 50 & 50 & 44,4 & 44,4 & 33,3 & 50 & 50 & 53,8 \\
\hline & 2017 & 9 & 0 & 11,1 & 55,6 & 22,2 & 22,2 & 11,1 & 11,1 & 11,1 & 20,0 \\
\hline & 2018 & 6 & 100 & 100 & 100 & 100 & 100 & 75 & 75 & 100 & 100,0 \\
\hline & 2019 & 5 & & 100 & 100 & 100 & 100 & 100 & 100 & 100 & 100,0 \\
\hline
\end{tabular}

N: de aislamientos ATM: Aztreoman FEP: Cefepime CAZ: Ceftazidima CIP: Ciprofloxacina GEN: Gentamicina IMP: Imipenem MEM: Meropenem AK: Amicacina.

Sensible menos del $30 \%$ 
En la Tabla 5 se reporta la sensibilidad intermedia para Aztreonam con una CMI50 variable, pero con tendencia a resistente, lo cual sería limitante para aquellos pacientes con antecedentes de alergia a B-lactámicos y carbapenémicos.

Tabla 5. Informe acumulado de susceptibilidad de la Pseudomona aeruginosa aislamientos en varios en pacientes del hospital.

\begin{tabular}{|c|c|c|c|c|c|c|c|c|c|c|c|}
\hline Año & $\mathbf{N}$ & ATM \%S & FEP \%S & CAZ \%S & CIP \%S & GEN \%S & IPM \%S & MEM \%S & TZP \%S & CT \%S & AK \%s \\
\hline 2015 & 136 & 50,5 & 73,7 & 75 & 61,6 & 63 & 75,8 & 67,4 & 62,8 & 99,26 & 87,7 \\
\hline 2016 & 97 & 57,1 & 71,7 & 84,7 & 67,5 & 77,9 & 70,1 & 73,8 & 61,7 & 100 & 85,2 \\
\hline 2017 & 121 & 47,8 & 68,8 & 78,9 & 57,4 & 75,2 & 66,7 & 72,5 & 69,4 & 100 & 86,2 \\
\hline 2018 & 92 & 51,8 & 75 & 74,2 & 71,6 & 81,8 & 66,3 & 72,1 & 75,3 & 100 & 87,6 \\
\hline 2019 & 85 & 45,5 & 85,7 & 87,5 & 74,1 & 73,2 & 83 & 90,4 & 80,4 & 100 & 95,5 \\
\hline
\end{tabular}

N: de aislamientos ATM: Aztreoman FEP: Cefepime CAZ: Ceftazidima CIP: Ciprofloxacina GEN: Gentamicina IMP: Imipenem MEM: Meropenem AK: Amicacina.

Sensible menos del $30 \%$ 
Aunque se identificó la presencia de una MBL por métodos fenotípicos en $P$. aeruginosa; se sugiere que los casos en los que se presentó resistencia a los carbapenémicos fueron debido a otros mecanismos; entre estos destaca la impermeabilidad de membrana, afectando principalmente a imipenem dentro de esta familia de antimicrobianos como se reporta en la Tabla 6.

Tabla 6. Identificación fenotípica de carbapenemasas.

\begin{tabular}{lllll}
\hline \multicolumn{1}{c}{ Pruebas } & \multicolumn{2}{c}{$\mathbf{2 0 1 8}(\mathbf{n}=\mathbf{2 9})$} & \multicolumn{2}{c}{$\mathbf{2 0 1 9}(\mathbf{n = 1 9 )}$} \\
\hline Inactivación carbapenémica $\left(a^{\star}\right)$ & + & - & + & - \\
Sinergia EDTA / $\left(b^{\star}\right)$ & 0 & 29,0 & 0 & 19,0 \\
Sinergia Caz+ Imp $\left(c^{\star}\right)$ & 0 & 29,0 & 1 & 18,0 \\
\hline
\end{tabular}

$\left(a^{\star}\right)$ Detección de Carbapenemasa / $\left(b^{\star}\right)$ Detección de Metabobelatactasamsa $\left(c^{\star}\right)$ Betalactamasas tipo GES.

\section{Discusión}

En este estudio la frecuencia en el sexo masculino fue predominante igual al reportado en Venezuela (25), Italia (26); sin embargo existen estudios que muestran resultados contrarios como en Teherán (Irán) (2). En cuanto a la incidencia de $P$. aeruginosa es comparable con la encontrada en otros países Ghana (27), Egipto (7), Vietnam (28); Italia (26), y la reportada en algunos países de América latina (29).

El mayor porcentaje de aislamiento provienen del servicio de hospitalización, puesto que el presente estudio se incluyeron las salas de cirugía, clínica, ginecobstetricia y quirófano, sin embargo, muestra relación con los reportados en Venezuela (25), Bolivia (30), Yemen (31), Egipto (7) en donde las secreciones o heridas fueron más representativas.

Respecto al perfil de resistencia a carbapenémicos Wang y colaborares(15), mostraron una prevalencia alta $29,2 \%$ en elárea de UCl, similar a la encontrada en este estudio, pues gran parte de las muestras ingresadas en esta Unidad son de MDR a XDR; mientras que Farhan y colaboradores (7) encontraron una alta incidencia de $\mathrm{MBL}$ en el área de $\mathrm{UCl}$, hecho que diferencia de este estudio. Por métodos fenotípicos se identificó únicamente una cepa MBL procedente de hospitalización en una secreción de herida, la mayor población de cepas XDR se concentraron en este mismo servicio, esto se debe a la existencia de una sala de aislamiento de infectología mismo que se encuentra dentro del servicio de hospitalización clínica.

La terapia antimicrobiana inicial con la Pseudomona aeruginosa es compleja pues se toma en cuenta varios factores y su rápida adaptación ha provocado mayor mortalidad; un antibiótico de primera línea por su acción antipseudomona podría ser piperacilina- 
tazobactam; sin embargo, en China (15) mostró una resistencia alta $>25 \%$ igual a la reportada en Pakistán (32), contrario al hallazgo en este estudio, pues la resistencia más alta no superó este valor y se manifiesta con una tendencia en descenso. Con relación a las cefalosporinas, se encontraron porcentaje son menores con respecto a los reportados en Lima-Perú del $37 \%$ (33), Sucre-Bolivia fue del 42,7\% (30), siendo mayores a los encontrados en Colombia (34) con una tendencia en descenso para ceftazidima comparable con China (15).

En cuanto al uso de ciprofloxacina el panorama es bueno pues se mantuvo estable a pesar de que los patrones encontrados difieren de otros países como, Ghana (27) o Pakistán (32).

Finalmente en cuanto a los antibióticos carbapenémicos imipenem resultó ser más susceptible, similar con Irán (2); contrario al que se manifiesta en Vietnam (28). Considerando el uso de terapia combinada, aminoglucósidos y polimixinas se encontró alta sensibilidad de colistina, hallazgo que se debe tener muy en cuenta y evitar la pérdida de sensibilidad y aparición de resistencia como la reportada en Brasil (35).

\section{CONCLUSIONES}

La Pseudomona aeruginosa es considerada como crítica por su multirresistencia, en muchos países se encuentra entre el tercer y cuarto lugar siendo las MBL tipo VIM las más frecuentes. Sin embargo, el presente estudio reveló niveles de resistencia mucho menor que los reportados a nivel mundial, es muy probable que esto se deba una baja incidencia en comparación con otros bacilos Gram negativos, sin embargo, la unidad de cuidados intensivos muestra una alta prevalencia con cepas MDR y XDR.

El uso racional de los antibióticos es primordial, para mantener niveles de susceptibilidad adecuados en un germen como el estudiado, considerando el sitio de acción, tipo de infección, entre otros factores que podrían afectar el tratamiento del paciente, sin embargo, se podrían considerar para el tratamiento de $P$. aeruginosa en primera línea frente a una infección del tracto urinario, se podría sugerir un tratamiento con fluoroquinolonas (ciprofloxacina) o $\beta$-lactámicos como: ceftazidima, piperacilinatazobactamocefepime; pues duranteel periodo en estudio, no existen valores alarmantes en cuanto a la resistencia antibacteriana, pues gran parte de estos se encuentran aceptables, pero cuando esta infección se da durante la estancia hospitalaria se debe tener en cuenta de la alta probabilidad de que se trate de un fenotipo MDR.

Las infecciones nosocomiales son más complejas en su tratamiento, sin embargo, el uso de ceftazidima, cefepime y ciprofloxacina deberá contar con la respectiva vigilancia por la creciente resistencia el medio hospitalario con pacientes con riesgos de coinfección se debería considerar el uso de terapia combinada piperacilina/tazobactam y los carbapenémicos, siempre en combinación con los aminoglucósidos y manteniendo la 
vigilancia respectiva; en el presente estudio no se identificaron cepas PDR y colistina tiene alta sensibilidad.

\section{REFERENCIAS BIBLOGRAFICAS}

1. Ramos J-L, Filloux A. editores. Pseudomonas. [Internet]. 2007 ed. Vol. 5. Dordrecht: Springer Netherlands; 2007 [citado 27 de abril de 2021]. Disponible en: http://link.springer. com/10.1007/978-1-4020-6097-7

2. Hadavand F, Gachkar L, Amini M. High Prevalence of Drug-Resistant Gram-Negative Bacteria: A Hospital-Based Cross-Sectional Study in Tehran, in 2017. Arch Clin Infect Dis. 13 de enero de 2020; In Press.

3. Wisplinghoff $\mathrm{H}$. Pseudomonas spp., Acinetobacter spp. and Miscellaneous GramNegative Bacilli. En: Cohen J, Powderly WG, Opal SM, editores. Infectious Diseases (Fourth Edition) [Internet]. Elsevier; 2017 [citado 27 de abril de 2021]. p. 1579-1599.e2. Disponible en: https://www.sciencedirect.com/science/article/ pii/B9780702062858001817

4. Malhotra S, Hayes D, Wozniak DJ. Cystic Fibrosis and Pseudomonas aeruginosa: the Host-Microbe Interface. Clin Microbiol Rev [Internet]. 19 de junio de 2019 [citado 27 de abril de 2021];32(3). Disponible en: https://cmr. asm.org/content/32/3/e00138-18

5. Brindhadevi K, Lewis Oscar F, Mylonakis E, ShanmugamS, VermaTN, Pugazhendhi A. Biofilm and Quorum sensing mediated pathogenicity in Pseudomonas aeruginosa. Process Biochem. 1 de septiembre de 2020;96:49-57.

6. Denis J-B, Lehingue S, Pauly V, Cassir N, Gainnier $M$, Léone $M$, et al. Multidrug-resistant Pseudomonas aeruginosa and mortality in mechanically ventilated ICU patients. Am J Infect Control. 1 de septiembre de 2019;47(9):1059-64.

7. Farhan SM, Ibrahim RA, Mahran KM, Hetta HF, El-Baky RMA. Antimicrobial resistance pattern and molecular genetic distribution of metallo- $\beta$ lactamases producing Pseudomonas aeruginosa isolated from hospitals in Minia, Egypt. Infect Drug Resist. 16 de julio de 2019;12:2125-33.

8. Hernández A, Yagüe G, Vázquez EG, Simón $M$, Parrado LM, Canteras $M$, et al. Infecciones nosocomiales por Pseudomonas aeruginosa multiresistente incluido carbapenémicos: factores predictivos y pronósticos. Estudio prospectivo 2016-2017. Rev Esp Quimioter. abril de 2018;31(2):123-30.

9. Ferrer R, Shahrour $H$, Pitts $B$, Stewart PS, Sánchez-Gómez S, Martínez-de-Tejada G. A permeability-increasing drug synergizes with bacterial efflux pump inhibitors and restores susceptibility to antibiotics in multi-drug resistant Pseudomonas aeruginosa strains. Sci Rep. 5 de marzo de 2019;9(1):3452.

10. Yaghi J, Fattouh N, Akkawi C, El Chamy L, Maroun RG, Khalil G. Unusually High Prevalence of Cosecretion of Ambler Class A and B Carbapenemases and Nonenzymatic Mechanisms in Multidrug-Resistant Clinical Isolates of Pseudomonas aeruginosa in Lebanon. Microb Drug Resist Larchmt N. febrero de 2020;26(2):150-9.

11. Organizacion Mundial de la Salud. La OMS publica la lista de las bacterias para las que se necesitan urgentemente nuevos antibióticos [Internet]. [citado 28 de abril de 2021]. Disponible en: https://www.who.int/es/news/item/27-022017-who-publishes-list-of-bacteria-for-whichnew-antibiotics-are-urgently-needed

12. Oliveira DMPD, Forde BM, Kidd TJ, Harris PNA, Schembri MA, Beatson SA, et al. Antimicrobial Resistance in ESKAPE Pathogens. Clin Microbiol Rev [Internet]. 17 de junio de 2020 [citado 28 de abril de 2021];33(3). Disponible en: https://cmr. asm.org/content/33/3/e00181-19

13. Duval RE, Grare M, Demoré B. Fight Against Antimicrobial Resistance: We Always Need New Antibacterials but for Right Bacteria. Molecules [Internet]. 29 de agosto de 2019 
[citado 30 de abril de 2021];24(17). Disponible en: https://www.ncbi.nlm.nih.gov/pmc/ articles/PMC6749585/

14. Surveillance of antimicrobial resistance in Europe 2018 [Internet]. European Centre for Disease Prevention and Control. 2019 [citado 28 de abril de 2021]. Disponible en: https://www.ecdc.europa.eu/en/publicationsdata/surveillance-antimicrobial-resistanceeurope-2018

15. Wang J, Zhou M, Huang G, Guo Z, Sauser $J$, Metsini $A$, et al. Antimicrobial resistance in southern China: results of prospective surveillance in Dongguan city, 2017. J Hosp Infect. 1 de junio de 2020;105(2):188-96.

16. Zhang Y, Zhong Z-F, Chen S-X, Zhou D-R, Li $Z-K$, Meng $Y$, et al. Prevalence of healthcareassociated infections and antimicrobial use in China: Results from the 2018 point prevalence survey in 189 hospitals in Guangdong Province. Int J Infect Dis. 1 de diciembre de 2019;89:17984.

17. Centers for Disease Control and Prevention. Antimicrobial Resistance in ESKAPE Pathogens I Clinical Microbiology Reviews [Internet]. [citado 30 de abril de 2021]. Disponible en: https://cmr.asm.org/content/33/3/e00181-19. abstract

18. Shortridge D, Gales AC, Streit JM, Huband MD, Tsakris A, Jones RN. Geographic and Temporal Patterns of Antimicrobial Resistance in Pseudomonas aeruginosa Over 20 Years From the SENTRY Antimicrobial Surveillance Program, 1997-2016. Open Forum Infect Dis. 15 de marzo de 2019;6(Suppl 1):S63-8.

19. Walters MS, Grass JE, Bulens SN, Hancock EB, Phipps EC, Muleta D, et al. CarbapenemResistant Pseudomonas aeruginosa at US Emerging Infections Program Sites, 2015. Emerg Infect Dis. julio de 2019;25(7):1281-8.

20. Organizacion Mundial de la Salud. OMS. Infección por "Pseudomonas aeruginosa " resistente a los carbapenémicos - México [Internet]. WHO. World Health Organization; [citado 30 de abril de 2021]. Disponible en: http://www.who.int/csr/don/5-march-2019carbapenem-resistant-p-aeruginosa-mex/es/

21. Zarpellon MN, Viana GF, Mitsugui CS, Costa BB, Tamura NK, Aoki EE, et al. Epidemiologic surveillance of multidrug-resistant bacteria in a teaching hospital: A 3-year experience. Am J Infect Control. 1 de abril de 2018;46(4):387-92.

22. Ministerio de Salud Pública. Gacetas IASS y RAM - Ministerio de Salud Pública [Internet]. [citado 30 de abril de 2021]. Disponible en: https://www.salud.gob.ec/gacetas-iass-y-ram/

23. Campoverde SL, Zúñiga AC. Prevalencia puntual de infecciones asociadas a la atención de salud en el Hospital Vicente Corral Moscoso, Cuenca 2019. 19 de febrero de 2019 [citado 1 de junio de 2021]; Disponible en: http://dspace. ucuenca.edu.ec/handle/123456789/34014

24. Siavichay L, Eugenia M. Prevalencia y factores asociados a infecciones intrahospitalarias en el área de clínica del Hospital Vicente Corral Moscoso, Cuenca 2019. 19 de mayo de 2021 [citado 1 de junio de 2021]; Disponible en: http://dspace.ucuenca. edu.ec/handle/123456789/36191

25. Quijada Martínez P, Flores Carrero A, Labrador I, Araque M. Estudio clínico y microbiológico de la infección urinaria asociada a catéter, en los servicios de medicina interna de un hospital universitario venezolano. Rev Peru Med Exp Salud Publica. enero de 2017;34(1):52-61.

26. NataleA,Stelling J, MeledandriM, Messenger LA, D'Ancona F. Use of WHONET-SaTScan system for simulated real-time detection of antimicrobial resistance clusters in a hospital in Italy, 2012 to 2014. Eurosurveillance. 16 de marzo de 2017;22(11):30484.

27. Opintan JA, Newman MJ, Arhin RE, Donkor ES, Gyansa-Lutterodt M, Mills-Pappoe W. Laboratory-based nationwide surveillance of 
antimicrobial resistance in Ghana. Infect Drug Resist. 18 de noviembre de 2015;8:379-89.

28. Vu TVD, Do TTN, Rydell U, Nilsson LE, Olson L, Larsson M, et al. Antimicrobial susceptibility testing and antibiotic consumption results from 16 hospitals in Viet Nam: The VINARES project 2012-2013. J Glob Antimicrob Resist. 1 de septiembre de 2019;18:269-78.

29. Vega S, Dowzicky MJ. Antimicrobial susceptibility among Gram-positive and Gramnegative organisms collected from the Latin American region between 2004 and 2015 as part of the Tigecycline Evaluation and Surveillance Trial. Ann Clin Microbiol Antimicrob. 12 de julio de 2017;16(1):50.

30. Boutier G. MAPA EPIDEMIOLÓGICO DE RESISTENCIA ANTIMICROBIANA DE LOS AGENTES BACTERIANOS AISLADOS EN EL HOSPITAL SANTA BÁRBARA. Bio Sci. 31 de diciembre de 2018;1(2):1-12.

31. Al Haik W, Al Mahbashi A, Almahdi A, Mohamed M, Al Haddad A. Genotypic Characteristics of Clinical and Non-Clinical Isolates of Pseudomonas aeruginosa: Distribution of Different antibiogram Profiles and Molecular Typing. Jordan J Biol Sci. 9 de septiembre de 2016;9:185-94.

32. Samad A, Ahmed T, Rahim A, Khalil A, Ali I. Antimicrobial susceptibility patterns of clinical isolates of Pseudomonas aeruginosa isolated from patients of respiratory tract infections in a Tertiary Care Hospital, Peshawar. Pak J Med Sci. 2017;33(3):670-4.

33. Hernández C, Hercilla L, Mendo F, PérezLazo G, Contreras E, Ramírez E, et al. Programas de optimización del uso de antimicrobianos en Perú: Un acuerdo sobre lo fundamental. Rev Chil Infectol. octubre de 2019;36(5):565-75.

34. Ibañez Dosman JP, Salazar Ospina JD, Loaiza Betancurt S, Hernández Botero JS. Panorama de resistencia antimicrobiana de los aislamientos urinarios de pacientes adultos en los servicios de urgencias de Manizales, Caldas, durante el 2018. Infectio. 15 de abril de 2020;24(3):149-54.

35. Rossi F, Girardello R, Cury AP, Di Gioia TSR, Almeida Jr JN de, Duarte AJ da S, et al. Emergence of colistin resistance in the largest university hospital complex of São Paulo, Brazil, over five years. Braz J Infect Dis. febrero de 2017;21(1):98-101.

Conflicto de intereses: Ninguno declarado por la autora Financiación: Ninguna declarada por la autora Agradecimiento: Ninguno manifestado por la autora

\section{ACERCA DEL AUTOR}

Diana Verónica Barbecho Coraisaca. Laboratorio Clínico, Universidad de Cuenca. Microbiología, Hospital Vicente Corral Moscoso. Maestrante en Diagnóstico de Laboratorio Clínico y Molecular, Universidad Católica de Cuenca, Ecuador. 